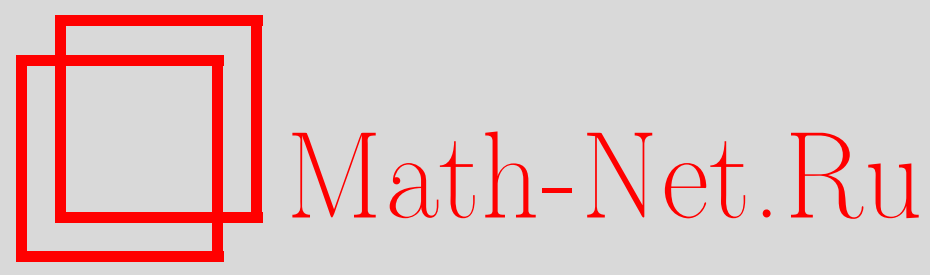

Е. Е. Золин, Секвенциальные рефлексивные логики с оператором разрешимости, Матем. заметки, 2002, том 72, выпуск 6, 853-868

DOI: https://doi.org/10.4213/mzm472

Использование Общероссийского математического портала Math-Net.Ru подразумевает, что вы прочитали и согласны с пользовательским соглашением http://www . mathnet.ru/rus/agreement

Параметры загрузки:

IP: 52.205 .19 .152

26 апреля 2023 г., $14: 16: 42$

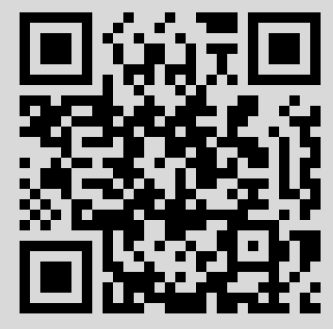




\title{
СЕКВЕНЦИАЛЬНЫЕ РЕФЛЕКСИВНЫЕ ЛОГИКИ С ОПЕРАТОРОМ РАЗРЕШИМОСТИ
}

\author{
Е. Е. Золин
}

\begin{abstract}
Построены системы гильбертовского типа $L^{\triangleright}$ и секвенциальные исчисления $\left[L^{\triangleright}\right]$ для вариантов логик $L=\mathbf{T}, \mathbf{S 4}, \mathbf{B}, \mathbf{S 5}$ и $\mathbf{G r z}$, сформулированных в языке с единственным модальным оператором разрешшмости $\triangleright A=\square A \vee \square \neg A$. Доказано, что в исчислениях $\left[L^{\triangleright}\right]$ сечение не устранимо, однако можно ограничиться аналитическим сечением, сохраняющим свойство подформульности. Тем самым, исчисления $\left[\mathbf{T}^{\triangleright}\right]$, $\left[\mathbf{S} \mathbf{4}^{\triangleright}\right],\left[\mathbf{S 5}^{\triangleright}\right]$ (соответственно $\left[\mathbf{G r z}^{\triangleright}\right]$ ) обладают (соответственно слабым) свойством подформульнсти (для $\left[\mathbf{B}_{2}^{\triangleright}\right]$ этот вопрос остается открытым). Установлено интерполяционное свойство Крейга для рассмотренных логик разрешимости.

Библиография: 17 названий.
\end{abstract}

Введение. При построении логических исчислений в модальной логике традиционным стал выбор языка с операторами необходимости $\square$ (и возможности $\diamond)$. Однако определенньй технический и философский интерес (см. [1], [2]) представляют системы, где в качестве базисного выбирается оператор разрешимости (или неслучайности), определяемьй равенством $\triangleright A=\square A \vee \square \neg A$. (Термин “неслучайность" (non-contingency) принят в англоязычной литературе; мы будем употреблять термин "разрешимость", происходящий из рассмотрения доказуемостной интерпретации оператора $\square:$ предложение разрешимо в теории, если в ней доказуемо либо оно, либо его отрицание). Этим равенством задается перевод $\triangleright$-формул (т.е. формул модального язька с единственным модальньм оператором $\triangleright$ или, иначе говоря, $\triangleright$-языка) в $\square$-формулы. Если задана $\square$-логика $L$ (т.е. логика в $\square$-язьке), то логикой разрешимости над $L$ (обозначение: $L^{\triangleright}$ ) назьвается множество $\triangleright$-формул, переводы которых являются теоремами $L$.

В работах [2], [3] были предложены различные аксиоматики логик разрешимости над известными нормальными логиками T, $\mathbf{S 4}$ и $\mathbf{S 5}$ (см. также [4], [5]). Отметим, что в случае, когда логика $L$ содержит $\mathbf{T}$, а точнее, аксиому рефлексивности $\square A \rightarrow A$, исследование логики $L^{\triangleright}$ упрощается благодаря тому, что оператор $\square$ вырази́м через $\triangleright$ посредством равенства $\square A=A \& \triangleright A$. Как следствие, построение гильбертовских аксиоматик этих логик $L^{\triangleright}$ становится автоматическим (см. лемму 4.5 настоящей работы) и потому не представляет большого интереса. Напротив, построение для $L^{\triangleright}$ секвенщиальных исчислений, обладаюших “хорошими” структурными свойствами (устранимость сечения,

Работа выполнена при частичной поддержке Российского фонда фундаментальных исследований (грант научной школы). 
свойство подформульности и т.п.), имеет определенный смысл. В статье [6] построен нетривиальньй пример логики, не содержащей $\mathbf{T}$, в которой, тем не менее, $\square$ вырази́м через $\triangleright$.

Систематическое изучение логики разрешимости было начато в работе [7], содержащей первую, достаточно громоздкую аксиоматику минимальной логики разрешимости (т.е. логики $\mathbf{K}^{\triangleright}$ ). В последующей работе [8] она была упрощена, а также была аксиоматизирована логика $\mathbf{K} \mathbf{4}^{\triangleright}$. В [9] предложена аксиоматика логики разрешимости над “эпистемической" логикой KD45; кроме того, для некоторых аксиом логик разрешимости были найдены элементарные эквиваленты. Наконец, в [10] аксиоматизирована логика $\mathbf{G L}^{\triangleright}$ и построены секвенциальные исчисления для $\mathbf{K}{ }^{\triangleright}, \mathbf{K} \mathbf{4}^{\triangleright}$ и $\mathbf{G} \mathbf{L}^{\triangleright}$.

Настоящая работа является продолжением этого цикла исследований. После формулировки необходимых определений (п. 1) мы представляем в п. 2 гильбертовские аксиоматики $L^{\triangleright}$ и секвенциальные исчисления $\left[L_{1}^{\triangleright}\right]$ и $\left[L_{2}^{\triangleright}\right]$ для логик разрешимости над $L \in\{\mathbf{T}, \mathbf{S 4}, \mathbf{B}, \mathbf{S 5}, \mathbf{G r z}\}$. В п. 3 описан метод доказательства полноты секвенциальных исчислений в $\triangleright$-языке с аналитическим сечением. Доказательству полноты построенных аксиоматик посвящен п. 4. В построенных секвенщиальных исчислениях сечение не устранимо, что установлено в п. 5; тем не менее, из доказанной в п. 4 теоремы о полноте вытекает, что исчисления $\left[\mathbf{T}_{2}^{\triangleright}\right],\left[\mathbf{S} 4_{2}^{\triangleright}\right],\left[\mathbf{S 5}_{2}^{\triangleright}\right]$ (соответственно $[\mathbf{G r z}]$ ) обладают (соответственно слабым) свойством подформульности (для $\left[\mathbf{B}_{2}^{\triangleright}\right]$ вопрос остается открытым), а в п. 5 также установлено интерполящионное свойство Крейга для построенных логик разрешимости.

1. Определения и факты. Пропозициональньй модальный язык $(\square$-язык) содержит счетное множество переменных $\mathbb{P}=\left\{p_{0}, p_{1}, \ldots\right\}$, булевы связки $\perp$ (ложь), $\rightarrow$ (импликация) и одноместньй оператор $\square$. Другие связки вводятся как сокращения, в частности: $\neg A \leftrightharpoons A \rightarrow \perp, \diamond A \leftrightharpoons \neg \square \neg A$. Множество $\square$-формул Fm $\square$ определяется обычным образом. Минимальная нормальная логика $\mathbf{K}$ имеет следующие аксиомы и правила вывода (здесь $A[B / p]$ - результат подстановки в формулу $A$ формулы $B$ вместо всех вхождений переменной $p)$ :

( $(\stackrel{\square}{\square})$ - классические тавтологии в $\square$-языке,

$\left(\mathrm{A}_{\mathbf{K}}^{\square}\right)$ - дистрибутивность: $\square(p \rightarrow q) \rightarrow(\square p \rightarrow \square q)$,

$$
\text { (MP) } \frac{A \quad A \rightarrow B}{B}, \quad(\mathrm{Sub}) \frac{A}{A[B / p]}, \quad(\mathrm{Nec}) \frac{A}{\square A} \text {. }
$$

Нас будут интересовать следующие нормальные модальные логики:

$$
\begin{array}{rlrl}
\mathbf{T} & =\mathbf{K}+\left(A_{\mathbf{T}}^{\square}\right), & \mathbf{S 4}=\mathbf{T}+\left(A_{\mathbf{4}}^{\square}\right), \\
\mathbf{B} & =\mathbf{T}+\left(A_{\mathbf{B}}^{\square}\right), & \mathbf{S 5} & =\mathbf{T}+\left(A_{\mathbf{5}}^{\square}\right), \\
\mathbf{S 4 . 1} & =\mathbf{S} \mathbf{4}+\left(A_{\mathbf{1}}^{\square}\right), & \mathbf{G r z} & =\mathbf{K}+\left(A_{\mathbf{G}}^{\square}\right),
\end{array}
$$

где дополнительные аксиомы задаются формулами

$$
\begin{aligned}
& \left(\mathrm{A}_{\mathbf{T}}^{\square}\right) \text { - рефлексивность: } \square p \rightarrow p, \\
& \left(\mathrm{~A}_{\mathrm{B}}^{\square}\right) \text { - симметричность: } p \rightarrow \square \diamond p,
\end{aligned}
$$


$\left(\mathrm{A}_{4}^{\square}\right)$ - транзитивность: $\square p \rightarrow \square \square p$,

$\left(\mathrm{A}_{5}^{\square}\right)$ - евклидовость: $\diamond p \rightarrow \square \diamond p$,

$\left(\mathrm{A}_{1}^{\square}\right)$ - аксиома Маккинси: $\square \diamond p \rightarrow \diamond \square p$,

$\left(\mathrm{A}_{\mathbf{G}}^{\square}\right)$ - аксиома Гжегорчика: $\square(\square(p \rightarrow \square p) \rightarrow p) \rightarrow p$.

При этом для перечисленных здесь модальных логик выполнена следующая диаграмма вложений:

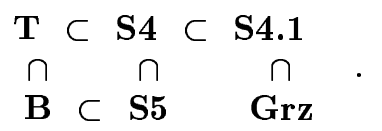

Секвениия есть выражение вида $\Pi \Rightarrow \Sigma$, где $\Pi$ и $\Sigma$ - конечные мультимножества ${ }^{1}$ формул. Включение мультимножеств формул мы понимаем без учета кратности, т.е. запись $\Pi \subseteq \Sigma$ означает, что всякая формула из П входит в $\Sigma$. Мы обозначаем $\Pi \Sigma:=\Pi \cup \Sigma$ и $\Pi A:=\Pi \cup\{A\}$. Множество подформул формулы $A$ обозначаем $\operatorname{Sb} A$, и если $\Gamma$ - (мульти)множество формул, то $\mathrm{Sb} \Gamma:=\cup\{\mathrm{Sb} A \mid A \in \Gamma\}$. Если секвенция П $\Rightarrow \Sigma$ обозначена как $w$, то обозначим ее антецедент через $\langle w|:=\Pi$, сукцедент через $|w\rangle:=\Sigma$, множество подформул $\mathrm{Sb} w:=\mathrm{Sb} \Pi \Sigma$. Пишем $A \in w$, если $A \in \Pi \Sigma$, также $\Gamma \subseteq w$, если $\Gamma \subseteq \Pi \Sigma$, и $w \subseteq \Gamma$, если П $\Sigma \subseteq \Gamma$. Если $\mathscr{L}$ - секвенциальное исчисление, то запись $\mathscr{L} \vdash A \Leftrightarrow B$ означает: $\mathscr{L} \vdash A \Rightarrow B$ и $\mathscr{L} \vdash B \Rightarrow A$.

Секвенциальное исчисление $[L]$ для логики

$$
L \in\{\mathbf{T}, \mathbf{S} 4, \mathbf{B}, \mathbf{S} 5, \mathbf{G r z}\}
$$

получается добавлением к секвенциальному исчислению высказываний (с сечением) правил $(\square \Rightarrow)$ и $\left(\Rightarrow_{L}^{\square}\right)$, задаваемых следующими формулами:

$$
\begin{array}{lll}
(\square \Rightarrow) \frac{A, \Pi \Rightarrow \Sigma}{\square A, \Pi \Rightarrow \Sigma}, & \left(\Rightarrow_{\mathbf{B}}^{\square}\right) \frac{\Pi \Rightarrow \square \Sigma, A}{\square \Pi \Rightarrow \Sigma, \square A}, \quad\left(\Rightarrow_{\mathrm{S} 5}^{\square}\right) \frac{\square \Pi \Rightarrow \square \Sigma, A}{\square \Pi \Rightarrow \square \Sigma, \square A}, \\
\left(\Rightarrow_{\mathbf{T}}^{\square}\right) \frac{\Pi \Rightarrow A}{\square \Pi \Rightarrow \square A}, & \left(\Rightarrow_{\mathrm{S} 4}^{\square}\right) \frac{\square \Pi \Rightarrow A}{\square \Pi \Rightarrow \square A}, \quad\left(\Rightarrow_{\mathbf{G} \mathbf{r z}}^{\square}\right) \frac{\square(A \rightarrow \square A), \square \Pi \Rightarrow A}{\square \Pi \Rightarrow \square A} .
\end{array}
$$

Известно, что сечение устранимо в исчислениях для T, S4 и $\mathbf{~ r r z ~ [ 1 1 ] ~ и ~ н е ~ у с т р а н и м о ~ в ~}$ исчислениях для $\mathbf{B}$ и $\mathbf{S 5}$ [12]-[14]. В последних можно ограничиться аналитическим сечением [15]:

$$
\frac{\Pi \Rightarrow \Sigma, A \quad A, \Pi^{\prime} \Rightarrow \Sigma^{\prime}}{\Pi \Pi^{\prime} \Rightarrow \Sigma \Sigma^{\prime}}, \quad A \in \mathrm{Sb}\left(\Pi \Pi^{\prime} \Sigma \Sigma^{\prime}\right) .
$$

Получающееся при этом исчисление [S5] обладает свойством подформульности [14]: всякая выводимая секвенция $\Pi \Rightarrow \Sigma$ имеет вывод, все секвенции которого состоят из подформул формул из П $\Sigma$. Правило $\left(\Rightarrow_{\mathrm{B}}^{\square}\right)$ может нарушать свойство подформульности, однако известно [14], что можно ограничиться такими его применениями, в которых $\Sigma \subseteq \mathrm{Sb}(\Pi A)$ и даже $\square \Sigma \subseteq \mathrm{Sb}(\Pi A)$. Тем самьм, свойство подформульности справедливо и для [B]. Наконец, исчисление [Grz] обладает слабымм свойством подформульности:

\footnotetext{
${ }^{1}$ Под мультимножеством понимается множество с указанием кратности $(\geqslant 0)$ вхождения элементов. Формально, мультимножество $\square$-формул есть отображение $\mathbf{F m} \square \rightarrow \mathbb{N}$.
} 
всякая вьводимая секвенция $\Pi \Rightarrow \Sigma$ имеет вывод, состоящий из секвенций вида $\Gamma \Rightarrow \Delta$,

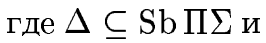

$$
\Gamma \subseteq \operatorname{Sb}(\Pi \Sigma \cup\{\square(A \rightarrow \square A) \mid \square A \in \mathrm{Sb} \Pi \Sigma\}) .
$$

Для формулировки логик разрешимости введем $\triangleright$-язык, отличающийся от $\square$-языка лишь заменой символа $\square$ на $\triangleright$, и множество $\triangleright$-формул $\mathbf{F m} \mathbf{m}^{\triangleright}$. Зададим $\triangleright$-перевод $\operatorname{tr}:$ $\mathbf{F m}^{\triangleright} \rightarrow \mathbf{F m}^{\square}$, сохраняющий переменные и булевы связки, такой, что

$$
\operatorname{tr}(\triangleright A)=\square \operatorname{tr}(A) \vee \square \neg \operatorname{tr}(A) .
$$

Вместо $\operatorname{tr}(A)$ будем часто писать $A_{\triangleright}$. Допуская вольность в обозначениях, мы иногда пишем $\triangleright A$, где $A$ есть $\square$-формула, подразумевая $\square A \vee \square \neg A$; такое использование символа $\triangleright$ легко распознать по контексту. Логикой разрешимости над логикой $L$ назовем множество $\triangleright$-формул, $\triangleright$-переводы которых являются теоремами логики $L$ :

$$
L^{\triangleright}:=\left\{A \in \mathbf{F m}^{\triangleright} \mid A_{\triangleright} \in L\right\} .
$$

Семантика Крипке для $\square$ - и $\triangleright$-языков вводится обычным образом. Отношение достижимости в шкале и обратное к нему мы будем обозначать $\uparrow$ и $\downarrow$ соответственно; кванторы по достижимым из $w$ точкам мы записьваем как $\forall x \downarrow w$ и $\exists x \downarrow w$. В этих обозначениях модальньй пункт определения истинности $\triangleright$-формулы в точке модели имеет вид

$$
w \models \triangleright A \leftrightharpoons(\forall x \downarrow w \quad x \models A) \text { или }(\forall x \downarrow w \quad x \not \models A) .
$$

Очевидно, что $w \models A \Leftrightarrow w \models A_{\triangleright}$ для любой $\triangleright$-формулы $A$. Если $\Gamma-$ множество формул, то Г-шкалой называется шкала, на которой общезначимо Г. Под истинностью (общезначимостью) секвенции $\Pi \Rightarrow \Sigma$ подразумеваем истинность (общезначимость) формулы $\bigwedge \Pi \rightarrow \bigvee \Sigma$.

2. Аксиоматические системы. Аксиомами минимальной рефлексивной логики разрешимости $\mathbf{T}^{\triangleright}$ являются все классические тавтологии в $\triangleright$-языке и следующие аксиомы:

$$
\begin{aligned}
& \left(\mathrm{A}_{\neg}^{\triangleright}\right) \text { - зеркальность: } \triangleright p \leftrightarrow \triangleright \neg p, \\
& \left(\mathrm{~A}_{\mathrm{T}}^{\triangleright}\right)-\text { слабая дистрибутивность: } p \rightarrow[\triangleright(p \rightarrow q) \rightarrow(\triangleright p \rightarrow \triangleright q)],
\end{aligned}
$$

а правилами вывода

$$
(\mathrm{MP}), \quad(\mathrm{Sub}) \text { и }(\mathrm{Dec}) \frac{A}{\triangleright A} .
$$

Аксиоматика других рефлексивных логик разрешимости приведена ниже (гипотеза: в формулировке исчисления $\mathbf{G r z}^{\triangleright}$ аксиома $\left(\mathrm{A}_{4}^{\triangleright}\right)$ является лишней):

$$
\begin{aligned}
\mathbf{B}^{\triangleright} & =\mathbf{T}^{\triangleright}+\left(\mathrm{A}_{\mathbf{B}}^{\triangleright}\right), & & \left(\mathrm{A}_{\mathbf{B}}^{\triangleright}\right)-\text { аксиома } p \rightarrow \triangleright(\triangleright p \rightarrow p), \\
\mathbf{S 4} & =\mathbf{T}^{\triangleright}+\left(\mathrm{A}_{\mathbf{4}}^{\triangleright}\right), & & \left(\mathrm{A}_{\mathbf{4}}^{\triangleright}\right)-\text { аксиома } \triangleright p \rightarrow \triangleright \triangleright p, \\
\mathbf{S} 5^{\triangleright} & =\mathbf{T}^{\triangleright}+\left(\mathrm{A}_{\mathbf{5}}^{\triangleright}\right), & & \left(\mathrm{A}_{\mathbf{5}}^{\triangleright}\right)-\text { аксиома } \triangleright \triangleright p, \\
\mathbf{G r z} & =\mathbf{S} \mathbf{4}^{\triangleright}+\left(\mathrm{A}_{\mathbf{G}}^{\triangleright}\right), & & \left(\mathrm{A}_{\mathbf{G}}^{\triangleright}\right)-\text { аксиома } \triangleright(\triangleright(p \rightarrow \triangleright p) \rightarrow p) \rightarrow \triangleright p .
\end{aligned}
$$

Далее $L$ обозначает одну из логик T, B, S4, S5, Grz. Записьвая выводы схематично, мы пишем

подразумевая

$$
L \vdash A_{0} \underline{1} A_{1} \underline{2} \cdots \underline{n} A_{n}, \quad \text { где } \underline{k} \in\{\rightarrow, \leftrightarrow\},
$$

$$
L \vdash A_{k-1} \stackrel{k}{A_{k}, \quad k=1, \ldots, n .}
$$


ЛЕмма 2.1. (а) Исчисления $L^{\triangleright}$ замкнуты относительно правила әквивалентной замены

$$
(\mathrm{RE}) \frac{A \leftrightarrow B}{\triangleright A \leftrightarrow \triangleright B}
$$

(б) Имеет место выводимость

$$
\mathbf{T}^{\triangleright} \vdash \triangleright p \& \triangleright q \rightarrow \triangleright(p \& q)
$$

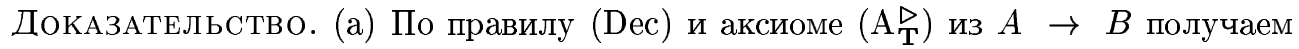
$A \rightarrow(\triangleright A \rightarrow \triangleright B)$, а из $\neg A \rightarrow \neg B$ сначала получим формулу $\neg A \rightarrow(\triangleright \neg A \rightarrow \triangleright \neg B)$, а затем - формулу $\neg A \rightarrow(\triangleright A \rightarrow \triangleright B)$ по аксиоме $\left(\mathrm{A}_{\neg}^{\triangleright}\right)$. Ввиду $\vdash A \vee \neg A$ из двух полученных формул выводим $\triangleright A \rightarrow \triangleright B$. Обратная импликация доказывается аналогично.

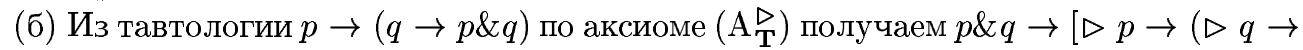
$\triangleright(p \& q))]$. Из тавтологии $\neg p \rightarrow \neg(p \& q)$ по аксиомам $\left(\mathrm{A}_{\text {T }}^{\triangleright}\right),\left(\mathrm{A}_{\neg}^{\triangleright}\right)$ получаем $\neg p \rightarrow[\triangleright p \rightarrow$ $\triangleright(p \& q)]$ и, тем более, $\neg p \rightarrow[\triangleright p \rightarrow(\triangleright q \rightarrow \triangleright(p \& q))]$. Аналогично вьводится $\neg q \rightarrow[\triangleright p \rightarrow(\triangleright q \rightarrow \triangleright(p \& q))]$. Наконец, в силу тавтологии $(p \& q) \vee \neg p \vee \neg q$ заключаем, что $\triangleright p \rightarrow(\triangleright q \rightarrow \triangleright(p \& q))$.

Для каждой из рассматриваемых логик $L$ сформулируем два секвенциальных исчисления $\left[L_{1}^{\triangleright}\right]$ и $\left[L_{2}^{\triangleright}\right]$. Исчисление $\left[L_{1}^{\triangleright}\right]$ получается добавлением к секвенциальному исчислению высказьваний (с сечением) правил $(\unrhd \Rightarrow),(\Rightarrow \unrhd)$ и $\left(\Rightarrow_{L}^{\triangleright}\right)$, задаваемых следующим образом:

$$
\begin{aligned}
& \left(\triangleright^{\triangleright} \Rightarrow\right) \frac{\triangleright A, \Pi \Rightarrow \Sigma}{\triangleright \neg A, \Pi \Rightarrow \Sigma}, \quad(\Rightarrow \underset{\mathrm{T}}{\triangleright}) \frac{\Pi \Rightarrow A}{\Pi, \triangleright \Pi \Rightarrow \triangleright A}, \quad\left(\Rightarrow_{\mathrm{B}}^{\triangleright}\right) \frac{\Pi \Rightarrow(\triangleright \Sigma \& \Sigma), A}{\Pi, \triangleright \Pi \Rightarrow \Sigma, \triangleright A}, \\
& (\Rightarrow \stackrel{\triangleright}{\triangleright}) \frac{\Pi \Rightarrow \Sigma, \triangleright A}{\Pi \Rightarrow \Sigma, \triangleright \neg A}, \quad\left(\Rightarrow_{\mathrm{S} 4}^{\triangleright}\right) \frac{\Pi, \triangleright \Pi \Rightarrow A}{\Pi, \triangleright \Pi \Rightarrow \triangleright A}, \quad\left(\Rightarrow \mathrm{S}_{5}\right) \frac{\Pi, \triangleright \Pi \Rightarrow \triangleright \Sigma, A}{\Pi, \triangleright \Pi \Rightarrow \triangleright \Sigma, \triangleright A}, \\
& \left(\Rightarrow \nabla_{\mathbf{G r z}}\right) \frac{\triangleright(A \rightarrow \triangleright A), \Pi, \triangleright \Pi \Rightarrow A}{\Pi, \triangleright \Pi \Rightarrow \triangleright A} .
\end{aligned}
$$

При формулировке правила $(\Rightarrow \underset{B}{\triangleright})$ использовано обозначение

$$
(\triangleright \Sigma \& \Sigma):=\{(\triangleright \sigma \& \sigma) \mid \sigma \in \Sigma\}
$$

Правила $(\stackrel{\neg}{\triangleright})$ и $(\Rightarrow \unrhd)$ нарушают свойство подформульности. Введем исчисление $\left[L_{2}^{\triangleright}\right]$, в котором данные правила поглощены другими. Это исчисление получаем добавлением к секвенциальному исчислению высказьваний (с сечением) правил $\left(\Rightarrow_{L}^{\triangleright r}\right), r \in\{0,1\}$, задаваемьх следующим образом:

$$
\begin{aligned}
& (\Rightarrow \underset{\mathbf{T}}{\triangleright r}) \frac{A^{\bar{r}}, \Pi \Rightarrow \Lambda, A^{r}}{\Pi, \triangleright(\Pi \Lambda) \Rightarrow \Lambda, \triangleright A}, \\
& (\Rightarrow \underset{\mathbf{B}}{\triangleright r}) \frac{\left\{A^{\bar{r}}, \Pi, \Phi^{\prime} \Rightarrow \Phi, \triangleright\left(\Psi^{\prime} \Psi\right), \Lambda, A^{r}\right\}_{\Sigma^{\prime}=\Phi^{\prime} \Psi^{\prime}}^{\Sigma=\Phi}}{\Pi, \triangleright(\Pi \Lambda), \Sigma^{\prime} \Rightarrow \Sigma, \Lambda, \triangleright A}, \\
& (\Rightarrow \mathrm{S} 4) \frac{A^{\bar{r}}, \Pi, \triangleright(\Pi \Lambda) \Rightarrow \Lambda, A^{r}}{\Pi, \triangleright(\Pi \Lambda) \Rightarrow \Lambda, \triangleright A}, \\
& \left(\Rightarrow \underset{\mathbf{G r z}}{\triangleright_{\mathbf{r}}}\right) \frac{A, \triangleright(A \vee \triangleright A), \Pi, \triangleright(\Pi \Lambda) \Rightarrow \Lambda}{\Pi, \triangleright(\Pi \Lambda) \Rightarrow \Lambda, \triangleright A}, \\
& (\Rightarrow \underset{\mathrm{S} 5}{\triangleright r}) \frac{A^{\bar{r}}, \Pi, \triangleright(\Pi \Lambda) \Rightarrow \Lambda, \triangleright \Sigma, A^{r}}{\Pi, \triangleright(\Pi \Lambda) \Rightarrow \Lambda, \triangleright \Sigma, \triangleright A}, \quad\left(\Rightarrow_{\mathbf{G r z}}^{\triangleright 1}\right) \frac{\triangleright(A \rightarrow \triangleright A), \Pi, \triangleright(\Pi \Lambda) \Rightarrow \Lambda, A}{\Pi, \triangleright(\Pi \Lambda) \Rightarrow \Lambda, \triangleright A} ;
\end{aligned}
$$


при их формулировке используются обозначения: $\bar{r}:=1-r, A^{0}:=\varnothing, A^{1}:=A$. Правила $\left(\Rightarrow{ }_{\mathbf{B}}^{r}\right), r \in\{0,1\}$, имеют $2^{|\Sigma|+\left|\Sigma^{\prime}\right|}$ посылок, отвечающих всевозможным разбиениям мультимножеств $\Sigma=\Phi \Psi$ и $\Sigma^{\prime}=\Phi^{\prime} \Psi^{\prime}$.

В п. 4 мы докажем, что в построенных исчислениях $\left[L_{k}^{\triangleright}\right]$ сечение не устранимо. Обозначим теперь через $\left[L_{2}^{\triangleright}\right]^{-}$исчисления, полученные из $\left[L_{2}^{\triangleright}\right]$ заменой правила сечения на аналитическое сечение. Как будет следовать из теоремы 4.1 о полноте, исчисления $\left[L_{2}^{\triangleright}\right]^{-}$и $\left[L_{2}^{\triangleright}\right]$ эквивалентны. Поэтому справедливо следующее утверждение.

Лемма 2.2. (а) Исчисления $\left[L_{2}^{\triangleright}\right]$, әде $L \in\{\mathbf{T}, \mathbf{S 4 , S 5}\}$, обладают свойством подформульности.

(б) Исчисление [Grz豆] обладает слабым свойством подформульности: всякая выводимая секвениия $\Pi \Rightarrow \Sigma$ имеет вывод, состоящий из секвенций вида $\Gamma \Rightarrow \Delta$, əде $\Delta \subseteq \operatorname{Sb} \Sigma u$

$$
\Gamma \subseteq \operatorname{Sb}(\Pi \Sigma \cup\{\triangleright(A \rightarrow \triangleright A), \triangleright(A \vee \triangleright A) \mid \triangleright A \in \operatorname{Sb} \Pi \Sigma\})
$$

В дальнейшем нам потребуется следующая

Лемма 2.3. (а) Для $L \in\{\mathbf{T}, \mathbf{S 4}, \mathbf{B}, \mathbf{S 5}, \mathbf{G r z}\}$ исчисление $\left[L_{1}^{\triangleright}\right]$ замкнуто относительно правила (RE), т.е. из $\left[L_{1}^{\triangleright}\right] \vdash A \Leftrightarrow B$ следует $\left[L_{1}^{\triangleright}\right] \vdash \triangleright A \Leftrightarrow \triangleright B$.

(б) Имеет место выводимость [ $\left.\mathbf{T}_{2}^{\triangleright}\right]^{-} \vdash \triangleright(p \rightarrow \triangleright p), p \Rightarrow \triangleright p$.

(в) Имеет место выводимость $\left[\mathbf{T}_{2}^{\triangleright}\right]^{-} \vdash \triangleright(p \vee \triangleright p) \Rightarrow p, \triangleright p$.

ДокАЗАТЕЛЬСТво. (а) Имеем вывод в $\left[\mathbf{T}_{1}^{\triangleright}\right]$ :

$$
\begin{array}{cl}
\frac{A \Rightarrow B}{A, \triangleright A \Rightarrow \triangleright B} & \frac{B \Rightarrow A}{\neg A \Rightarrow \neg B} \\
\frac{\triangleright A \Rightarrow \triangleright B, \neg A, \quad \neg A, \triangleright \neg A \Rightarrow \triangleright \neg B}{\triangleright A, \triangleright \neg A \Rightarrow \triangleright B, \triangleright \neg B .}
\end{array}
$$

Применив сечения с секвенцией $\triangleright A \Rightarrow \triangleright \neg A$ (по формуле $\triangleright \neg A$ ) и с секвенцией $\triangleright \neg B \Rightarrow$ $\triangleright B$ (по формуле $\triangleright \neg B$ ), а затем сокращения, получим $\triangleright A \Rightarrow \triangleright B$. Обратная секвенция доказьвается аналогично.

(б) Пользуясь аналитическим сечением, вьводим $\left[\mathbf{T}_{2}^{\triangleright}\right]^{-}$:

$$
\begin{aligned}
& \frac{p \Rightarrow \triangleright p, p}{\frac{p(p \rightarrow \triangleright p), p}{(p \rightarrow \triangleright} \quad \frac{p \Rightarrow p \quad \triangleright p \Rightarrow \triangleright p}{(p \rightarrow \triangleright) \Rightarrow(p \rightarrow \triangleright p), \triangleright p \quad(p \rightarrow \triangleright p), \quad p \Rightarrow \triangleright p}} \\
& \frac{\triangleright(p \rightarrow \triangleright p), p \Rightarrow \triangleright p, \triangleright p}{\triangleright(p \rightarrow \triangleright p), p \Rightarrow \triangleright p .}
\end{aligned}
$$

(в) Аналогично пункту (б).

3. Метод замыкания. В этом пункте описан метод доказательства полноты для произвольного непротиворечивого секвенциального исчисления $\mathscr{L}$ (в $\triangleright$-языке) с аналитическим сечением. 
ОПРЕДЕЛЕНИЕ 3.1. Множество формул Г замкнуто, если $\mathrm{Sb} \Gamma \subseteq \Gamma$. Секвенщию $w$ назовем замкнутой, если $\mathrm{Sb} w \subseteq w$, т.е. всякая подформула формулы из $w$ содержится в антецеденте или сукцеденте секвенции $w$; секвенцию $w$ назовем тонкой, если ее антецедент и сукцедент являются множествами, т.е. формулы в них не повторяются.

Очевидно, для всякого конечного (мульти)множества формул существует наименьшее содержащее его конечное замкнутое множество. Построим конечную шкалу $F_{\mathscr{L}}^{\Gamma}:=$ $\left(W_{\mathscr{L}}^{\Gamma}, \uparrow\right)$ и модель $M_{\mathscr{L}}^{\Gamma}:=\left(F_{\mathscr{L}}^{\Gamma}, \models\right)$, где $\Gamma \neq \varnothing$ - конечное замкнутое множество формул. Множество

$$
W_{\mathscr{L}}^{\Gamma}:=\{w \subseteq \Gamma \mid w \text { есть замкнутая тонкая секвенция, } \mathscr{L} \nvdash w\},
$$

очевидно, конечно.

ЛЕмма 3.2 (О замыкании). Всякую невыводимую в $\mathscr{L}$ секвенцию $\Pi \Rightarrow \Sigma$, состоящую из формул множества $\Gamma$, можсно расширить до тонкой замкнутой невыводимой в $\mathscr{L}$ секвенции. Формально, если $\Pi \subseteq \Gamma и \mathscr{L} \nvdash \Pi \Rightarrow \Sigma$, то

$$
\exists w \in W_{\mathscr{L}}^{\Gamma}: \Pi \subseteq\langle w|, \Sigma \subseteq| w\rangle
$$

ДокАЗАТЕЛЬСТво проводится стандартным методом замыкания: если $\mathscr{L} \nvdash \Pi \Rightarrow \Sigma$, $A \notin \Pi \Sigma$ и $A \in \mathrm{Sb} \Pi \Sigma$, то ввиду наличия в $\mathscr{L}$ аналитического сечения (и сокращения) имеем $\mathscr{L} \nvdash \Pi \Rightarrow \Sigma A$ или $\mathscr{L} \nvdash A \Pi \Rightarrow \Sigma$, поэтому $A$ можно добавить в антецедент или сукцедент секвенции $\Pi \Rightarrow \Sigma$. Процесс продолжается, пока секвенция $\Pi \Rightarrow \Sigma$ не станет замкнутой.

Отметим, что ввиду $\Gamma \neq \varnothing$ имеем $\perp \in \Gamma$ или $p \in \Gamma$ для некоторой переменной $p$. Значит, Г содержит секвенцию $\Rightarrow \perp$ или $\Rightarrow p$, очевидно, не вьводимую в $\mathscr{L}$. По лемме о замькании ее можно погрузить в некоторый "мир" $w \in W_{\mathscr{L}}^{\Gamma}$. Таким образом, $W_{\mathscr{L}}^{\Gamma} \neq \varnothing$.

Зададим оценку переменных, положив

$$
w \models p \leftrightharpoons p \in\langle w| \quad \text { для любых } w \in W_{\mathscr{L}}^{\Gamma} \quad \text { и } p \in \mathbb{P} .
$$

Осталось задать отношение $\uparrow$. Сформулируем условие на $\uparrow$, вьполнения которого достаточно для наших целей:

$$
\forall w \in W_{\mathscr{L}}^{\Gamma} \forall A \in w \quad w \models A \Leftrightarrow A \in\langle w| .
$$

ЛЕмма 3.3. Если выполнено $\left\langle 1^{\triangleright}\right\rangle$, то для любих $\Pi \Sigma \subseteq \Gamma$ из $\mathscr{L} \nvdash \Pi \Rightarrow \Sigma$ следует $M_{\mathscr{L}}^{\Gamma} \not=\Pi \Rightarrow \Sigma$.

ДокАЗАТЕЛЬСТво. По лемме о замыкании

$$
\Pi \subseteq\langle w| \quad \text { и } \quad \Sigma \subseteq|w\rangle
$$

для некоторого $w \in W_{\mathscr{L}}^{\Gamma}$. По $\left\langle 1^{\triangleright}\right\rangle$ имеем $w \models \wedge \Pi$ и $w \models \wedge \neg \Sigma$, т.е. $w \not \models \Pi \Rightarrow \Sigma$.

Покажем далее, что для удовлетворения $\left\langle 1^{\triangleright}\right\rangle$ достаточно наложить на $\uparrow$ следующее условие (квадратная скобка означает дизъюнкцию условий):

$$
\forall w \in W_{\mathscr{L}}^{\Gamma} \forall \triangleright B \in w \quad \triangleright B \in\langle w| \Leftrightarrow\left[\begin{array}{lll}
\forall x \downarrow w & B \in\langle x|, \\
\forall x \downarrow w & B \in|x\rangle .
\end{array}\right.
$$


Лемма 3.4. Имеет место импликация $\left\langle 2^{\triangleright}\right\rangle \Longrightarrow\left\langle 1^{\triangleright}\right\rangle$.

ДокАЗАТЕЛЬСтво проведем одновременно для всех $w \in W_{\mathscr{L}}^{\Gamma}$ индукцией по построению формулы $A \in w$. При $A \equiv \perp$ левая и правая части $\left\langle 1^{\triangleright}\right\rangle$ ложны. Для $A \equiv p$ утверждение следует из определения $\mid=$.

Пусть $A \equiv(B \rightarrow C)$. Так как секвенция $w$ замкнута, то $B, C \in w$ и по предположению индукции

(b) $\quad w \models B \Leftrightarrow B \in\langle w|, \quad w \not \models B \Leftrightarrow B \in| w\rangle$;

(c) $\quad w \models C \Leftrightarrow C \in\langle w|, \quad w \not \neq C \Leftrightarrow C \in| w\rangle$.

Отсюда

$$
w \models(B \rightarrow C) \stackrel{\text { def }=}{\Longleftrightarrow}\left[\begin{array} { l } 
{ w \not = B } \\
{ w \models C }
\end{array} \stackrel { ( b , c ) } { \Longleftrightarrow } \left[\begin{array}{l}
B \in|w\rangle \\
C \in\langle w|
\end{array} \stackrel{(?)}{\Longleftrightarrow}(B \rightarrow C) \in\langle w| .\right.\right.
$$

Докажем эквивалентность, помеченную знаком вопроса (?).

$(\Rightarrow)$ Если $(B \rightarrow C) \in|w\rangle$, то $B \notin|w\rangle$ и $C \notin\langle w|$, поскольку в $\mathscr{L}$ доказуемы секвенции $\Rightarrow B,(B \rightarrow C)$ и $C \Rightarrow(B \rightarrow C)$.

$(\Leftarrow)$ Если $(B \rightarrow C) \in\langle w|$, то невозможно одновременное выполнение условий $B \in\langle w|$ и $C \in|w\rangle$, ибо секвенция $(B \rightarrow C), B \Rightarrow C$ доказуема в $\mathscr{L}$.

Наконец, пусть $A \equiv \triangleright B$. По предположению индукции для любого $x \in W_{\mathscr{L}}^{\Gamma}$ если $B \in x$, то

$$
\text { (x) } \quad x \models B \Leftrightarrow B \in\langle x|, \quad x| \neq B \Leftrightarrow B \in \mid x\rangle \text {. }
$$

Отсюда

$$
\begin{aligned}
& \triangleright B \in\langle w| \stackrel{\left\langle 2^{\triangleright}\right\rangle}{\Longrightarrow}\left[\begin{array} { l l l } 
{ \forall x \downarrow w } & { B \in \langle x | } \\
{ \forall x \downarrow w } & { B \in | x \rangle }
\end{array} \stackrel { ( \mathbf { x } ) } { \Longrightarrow } \left[\begin{array}{lll}
\forall x \downarrow w & x=B \\
\forall x \downarrow w & x \neq B
\end{array} \stackrel{\text { def }=}{\Longrightarrow} w \models \triangleright B,\right.\right. \\
& \triangleright B \in|w\rangle \stackrel{\left\langle 2^{\triangleright}\right\rangle}{\Longrightarrow}\left\{\begin{array} { l l } 
{ \exists x \downarrow w } & { B \in \langle x | } \\
{ \exists y \downarrow w } & { B \in | y \rangle }
\end{array} \stackrel { ( \mathbf { x } ) } { \Longrightarrow } \left\{\begin{array}{ll}
\exists x \downarrow w & x \mid=B \\
\exists y \downarrow w & y \not=B
\end{array} \stackrel{\text { def }=}{\Longrightarrow} w \not \models \triangleright B .\right.\right.
\end{aligned}
$$

Теперь полноту логики $\mathscr{L}$ относительно класса конечных шкал $\mathscr{F}$ можно доказывать следующим образом. Пусть $\mathscr{L} \forall \Pi \Rightarrow \Sigma$. Строим конечное замкнутое множество $\Gamma \supseteq \Pi \Sigma$ и отношение $\uparrow$ так, что $F_{\mathscr{L}}^{\Gamma} \in \mathscr{F}$ и выполнено условие $\left\langle 2^{\triangleright}\right\rangle$. Из него по лемме 3.4 вытекает $\left\langle 1^{\triangleright}\right\rangle$, и в силу леммы 3.3 получаем $F_{\mathscr{L}}^{\Gamma} \not \models \Pi \Rightarrow \Sigma$, что и требовалось.

4. Полнота аксиоматик. Доказьваемая в этом пункте теорема утверждает, что построенные выше гильбертовские и секвенциальные исчисления дают полную аксиоматизацию логик разрешимости над T, S4, B, S5 и Grz. В конце пункта мы аксиоматизируем логику $\mathbf{S 4 . 1 ^ { \triangleright }}$.

ТЕорема 4.1 (совместная теорема о полноте). Для каждой логики $L \in\{\mathbf{T}, \mathbf{S} 4, \mathbf{B}$, S5, Grz\} и любой секвениии $\Pi \Rightarrow \Sigma$ в $\triangleright$-языке әквивалентны следующие утверждения:

(1) $\left[L_{2}^{\triangleright}\right]^{-} \vdash \Pi \Rightarrow \Sigma$,

(2) $\left[L_{1}^{\triangleright}\right] \vdash \Pi \Rightarrow \Sigma$,

(3) $L^{\triangleright} \vdash \bigwedge \Pi \rightarrow \bigvee \Sigma$,

(4) $L \vdash(\bigwedge \Pi \rightarrow \bigvee \Sigma)_{\triangleright}$,

(5) $F \models \Pi \Rightarrow \Sigma$ для любой конечной $L$-шкаль $F$. 
ДокаЗАТЕЛЬСтво проведем по схеме $(1) \Longrightarrow(2) \Longrightarrow(3) \Longrightarrow(4) \Longleftrightarrow(5) \Longrightarrow(1)$. Импликация $(2) \Longrightarrow(3)$ доказывается индукцией по построению вывода в $\left[L_{1}^{\triangleright}\right]$; при этом шаги, отвечающие правилам ( $\Rightarrow)$ и $(\Rightarrow \unrhd)$, очевидны ввиду наличия в логиках $L^{\triangleright}$ аксиомы $\left(\mathrm{A}_{\neg}\right)$; поэтому необходимо лишь проверять шаги, отвечающие правилу $\left(\Rightarrow_{L}^{\triangleright}\right)$. Импликацию $(3) \Longrightarrow(4)$ достаточно доказьвать лишь для $\Pi=\varnothing$ и $\Sigma=\{A\}$, т.е. проверять выводимость $\triangleright$-переводов аксиом $L^{\triangleright}$ в $L$; для аксиомы ( $\left.\mathrm{A} \unrhd\right)$ эта проверка тривиальна, а для $\left(\mathrm{A}_{\mathrm{T}}^{\triangleright}\right),\left(\mathrm{A}_{4}^{\triangleright}\right)$ и $\left(\mathrm{A}_{5}^{\triangleright}\right)$ она проведена в [2], [3]. Далее, эквивалентность $(4) \Longleftrightarrow(5)$ есть известная (см. [16], [17]) теорема о полноте логик $L$. Наконец, при доказательстве импликации $(5) \Longrightarrow(1)$ мы обозначаем исчисление $\mathscr{L}:=\left[L_{2}^{\triangleright}\right]^{-}$.

$(1) \Longrightarrow(2)$ Достаточно показать, что правила $\left(\Rightarrow_{L}^{\triangleright r}\right)$ являются производньми в $\left[L_{1}^{\triangleright}\right]$. Выведем, например, заключение правила $\left(\Rightarrow_{\mathbf{T}}^{\triangleright 0}\right)$ из его посылки в исчислении $\left[\mathbf{T}_{1}^{\triangleright}\right]$ :

$$
\begin{gathered}
\frac{A, \Pi \Rightarrow \Lambda}{\neg \Lambda \Rightarrow \neg A} \\
\overline{\Pi, \neg \Lambda, \triangleright \Pi, \triangleright \neg \Lambda \Rightarrow \triangleright \neg A .} .
\end{gathered}
$$

Применив сечение с секвенцией $\triangleright \neg A \Rightarrow \triangleright A$ (по формуле $\triangleright \neg A$ ), а также с секвенциями $\Rightarrow C, \neg C$ (по $\neg C$ ) и $\triangleright C \Rightarrow \triangleright \neg C$ (по $\triangleright \neg C$ ) для всех $C \in \Lambda$, мы получим $\Pi, \triangleright(\Pi \Lambda) \Rightarrow \Lambda, \triangleright A$.

При рассмотрении правила $(\Rightarrow \underset{\mathbf{G r z}}{\triangleright 0})$ потребуется выводимость в $\left[\mathbf{G r z} \mathbf{1}_{1}^{\triangleright}\right]$ секвенции

$$
\triangleright(\neg A \rightarrow \triangleright \neg A) \Rightarrow \triangleright(A \vee \triangleright A),
$$

вытекающая из леммы 2.3 (a).

Логика T. (2) $\Longrightarrow$ (3) По лемме 2.1 (б) $\mathbf{T}^{\triangleright} \vdash \wedge \triangleright \Pi \rightarrow \triangleright \wedge$ П, поэтому выводим в $\mathbf{T}^{\triangleright}$ :

$$
\begin{gathered}
\frac{\bigwedge \Pi \rightarrow B}{\triangleright(\bigwedge \Pi \rightarrow B)} \\
\frac{\bigwedge \Pi \rightarrow[\triangleright \wedge \Pi \rightarrow \triangleright B]}{\bigwedge\{\Pi, \triangleright \Pi\} \rightarrow \triangleright B .}
\end{gathered}
$$

$(5) \Longrightarrow(1)$ Допустим $\mathscr{L} \not \forall \Pi \Rightarrow \Sigma$. Возьмем конечное замкнутое множество $\Gamma:=$ $\mathrm{Sb} \Pi \Sigma$. На $W_{\mathscr{L}}^{\Gamma}$ введем рефлексивное отношение

$$
w \uparrow x \leftrightharpoons \forall C \in \mathbf{F m}^{\triangleright} \quad \triangleright C \in\langle w| \Rightarrow\left\{\begin{array}{l}
C \in\langle w| \Rightarrow C \in\langle x|, \\
C \in|w\rangle \Rightarrow C \in|x\rangle .
\end{array}\right.
$$

Тогда $F_{\mathscr{L}}^{\Gamma}$ - конечная $\mathbf{T}$-шкала, и остается проверить условие $\left\langle 2^{\triangleright}\right\rangle$.

ЛЕмма 4.2. Имеет место импликаиия $\left\langle 3_{\mathrm{T}}^{\triangleright}\right\rangle \Longrightarrow\left\langle 2^{\triangleright}\right\rangle$.

ДокАЗАТЕльСтво. Докажем эквивалентность в $\left\langle 2^{\triangleright}\right\rangle$. Берем $w \in W_{\mathscr{L}}^{\Gamma}$ и $\triangleright B \in w$.

$(\Rightarrow)$ Пусть $\triangleright B \in\langle w|$. Ввиду замкнутости $w$ возможны два случая:

1) $B \in\langle w|$, тогда $\forall x \downarrow w$ по $\left\langle 3_{\text {T }}^{\stackrel{⿱}{\mathbf{T}}}\right\rangle$ получаем $B \in\langle x|$;

2) $B \in|w\rangle$, аналогично $\forall x \downarrow w$ получаем $B \in|x\rangle$. 
$(\Leftarrow)$ Пусть $\triangleright B \in|w\rangle$. Построим такие $x, y \downarrow w$, что $B \in\langle x, B \in \mid y\rangle$.

Случай $B \in|w\rangle$. Выбор $y$ очевиден: $y:=w$. Положим

$$
\Pi:=\{C \in\langle w|| \triangleright C \in\langle w|\}, \quad \Lambda:=\{C \in|w\rangle \mid \triangleright C \in\langle w|\} .
$$

Тогда $\mathscr{L} \nvdash B, \Pi \Rightarrow \Lambda$, ибо иначе по правилу $\left(\Rightarrow \underset{\text { т }}{\triangleright^{0}}\right.$ ) получим $\mathscr{L} \vdash \Pi, \triangleright(\Pi \Lambda) \Rightarrow \Lambda, \triangleright B$, откуда ослаблением $\mathscr{L} \vdash w$. По лемме о замыкании $\exists x \in W_{\mathscr{L}}^{\Gamma}: \Pi \subseteq\langle x|, B \in\langle x|, \Lambda \subseteq| x\rangle$. Докажем, что $w \uparrow x$. Пусть $\triangleright C \in\langle w|$. Если $C \in\langle w|$, то $C \in \Pi \subseteq\langle x|$; если же $C \in|w\rangle$, то $C \in \Lambda \subseteq|x\rangle$.

Случай $B \in\langle w|$. Положив $x:=w$ и используя $(\Rightarrow \underset{\mathbf{T}}{\stackrel{1}{1}}$ ), аналогично строим искомый $y$ (для остальньх логик мы будем обычно рассматривать только первьй случай). Лемма доказана.

Логика $\mathbf{S 4 . ~ ( 2 ) ~ \Longrightarrow ~ ( 3 ) ~ В ь в о д ~ в ~} \mathbf{S 4}{ }^{\triangleright}$ :

$$
\frac{\bigwedge\{\Pi, \triangleright \Pi\} \rightarrow B}{\frac{\bigwedge\{\Pi, \triangleright \Pi\} \rightarrow[\triangleright \bigwedge\{\Pi, \triangleright \Pi\} \rightarrow \triangleright B]}{\frac{\bigwedge\{\Pi, \triangleright \Pi, \triangleright \triangleright \Pi\} \rightarrow \triangleright B}{\bigwedge\{\Pi, \triangleright \Pi\} \rightarrow \triangleright B .}}}
$$

$(5) \Longrightarrow(1) \mathrm{Ha} W_{\mathscr{L}}^{\Gamma}$ введем рефлексивное транзитивное отношение

$$
w \uparrow x \leftrightharpoons \forall C \in \mathbf{F m}^{\triangleright} \quad \triangleright C \in\langle w| \Rightarrow \triangleright C \in\langle x| \&\left\{\begin{array}{l}
C \in\langle w| \Rightarrow C \in\langle x|, \\
C \in|w\rangle \Rightarrow C \in|x\rangle .
\end{array}\right.
$$

В доказательстве $\left\langle 2^{\triangleright}\right\rangle$ имеем $\mathscr{L} \nvdash B, \Pi, \triangleright(\Pi \Lambda) \Rightarrow \Lambda$, иначе по правилу $(\Rightarrow \underset{\mathrm{S} 4}{\triangleright 0})$ получим $\mathscr{L} \vdash w$. Для $x \in W_{\mathscr{L}}^{\Gamma}$ такого, что $\Pi, \triangleright(\Pi \Lambda) \subseteq\langle x|$ и $\Lambda \subseteq|x\rangle$, очевидно, что $w \uparrow x$.

Логика В. $(2) \Longrightarrow(3)$ Обозначая $\Omega:=\neg \Sigma$ и используя выводимые в $\mathbf{B}^{\triangleright}$ формулы $p \rightarrow(\triangleright p \rightarrow p)$ и $p \rightarrow \triangleright(\triangleright p \rightarrow p)$, строим вьвод в $\mathbf{B}^{\triangleright}$ :

$$
\begin{gathered}
\frac{\bigwedge \Pi \rightarrow \bigvee\{(\triangleright \Sigma \& \Sigma), B\}}{\bigwedge\{\Pi,(\triangleright \Omega \rightarrow \Omega)\} \rightarrow B} \\
\frac{\bigwedge\{\Pi, \triangleright \Pi,(\triangleright \Omega \rightarrow \Omega), \triangleright(\triangleright \Omega \rightarrow \Omega)\} \rightarrow \triangleright B}{\bigwedge\{\Pi, \triangleright \Pi, \Omega\} \rightarrow \triangleright B} \\
\frac{\bigwedge\{\Pi, \triangleright \Pi\} \rightarrow \bigvee\{\Sigma, \triangleright B\} .}{}
\end{gathered}
$$

$(3) \Longrightarrow(4)$ Вьведем $\triangleright$-перевод аксиомы $\left(\mathrm{A}_{\mathrm{B}}^{\triangleright}\right)$ в логике В. Имеем

$$
\begin{aligned}
\mathbf{B} \vdash p & \longrightarrow \square \diamond p \longleftrightarrow \square[\diamond p \&(\neg p \rightarrow \diamond \neg p)] \\
& \longleftrightarrow \square[p \vee(\diamond p \& \diamond \neg p)] \longleftrightarrow \square(p \vee \neg \triangleright p) \longrightarrow \triangleright(\triangleright p \rightarrow p) .
\end{aligned}
$$

$(5) \Longrightarrow(1) \mathrm{Ha} W_{\mathscr{L}}^{\Gamma}$ сначала введем рефолексивное отношение $\uparrow$ условием $\left\langle 3_{\mathrm{T}}^{\triangleright}\right\rangle$, а затем возьмем его симметризацию:

$$
w \Uparrow x \leftrightharpoons(w \uparrow x) \&(x \uparrow w)
$$


Для доказательства $\left\langle 2^{\triangleright}\right\rangle$ берем П и $\Lambda$ как вьше, а также

$$
\Sigma:=\{C \in|w\rangle|\triangleright C \in| w\rangle\}, \quad \Sigma^{\prime}:=\{C \in\langle w|| \triangleright C \in \mid w\rangle\} .
$$

Существует разбиение $\Sigma=\Phi \Psi, \Sigma^{\prime}=\Phi^{\prime} \Psi^{\prime}$ такое, что $\mathscr{L} \nvdash B, \Pi, \Phi^{\prime} \Rightarrow \Phi, \triangleright\left(\Psi^{\prime} \Psi\right), \Lambda$, иначе, исходя из всевозможных секвенций такого вида по правилу $\left(\Rightarrow \triangleright_{\mathrm{B}}^{0}\right)$, мы бы вывели ${ }^{2}$ секвенцию П, $\triangleright(\Pi \Lambda), \Sigma^{\prime} \Rightarrow \Sigma, \Lambda, \triangleright B$ и далее ослаблением $\mathscr{L} \vdash w$.

Осталось для $x \in W_{\mathscr{L}}^{\Gamma}$ такого, что $\Pi \Phi^{\prime} \subseteq\langle x|$ и $\Phi, \triangleright\left(\Psi^{\prime} \Psi\right), \Lambda \subseteq|x\rangle$, проверить $w \Uparrow x$. Заметим, что $x \subseteq w$. Условие $w \uparrow x$ проверяется как в случае логики $\mathbf{T}$. Докажем $x \uparrow w$. Пусть $\triangleright C \in\langle x|$. Тогда $\triangleright C \in w$ ввиду $x \subseteq w$, и $C \in w$ в силу замкнутости $w$.

Далее, пусть $C \in\langle x|$. Если бы $C \in|w\rangle$, то были бы возможны следующие случаи:

1) $\triangleright C \in\langle w|$, тогда $C \in \Lambda \subseteq|x\rangle$, что неверно;

2) $\triangleright C \in|w\rangle$, тогда $C \in \Sigma=\Phi \Psi$ и мы имеем: если $C \in \Phi$, то $C \in|x\rangle$, что неверно; если же $C \in \Psi$, то $\triangleright C \in \triangleright \Psi \subseteq|x\rangle$, что тоже неверно.

Теперь пусть $C \in|x\rangle$. Если бы $C \in\langle w|$, то были бы возможны следуюшие случаи:

1) $\triangleright C \in\langle w|$, тогда $C \in \Pi \subseteq\langle x|$, что неверно;

2) $\triangleright C \in|w\rangle$, тогда $C \in \Sigma^{\prime}=\Phi^{\prime} \Psi^{\prime}$ и мы имеем: если $C \in \Phi^{\prime}$, то $C \in\langle x|$, что неверно; если же $C \in \Psi^{\prime}$, то $\triangleright C \in \triangleright \Psi^{\prime} \subseteq|x\rangle$, что тоже неверно.

Логика S5. (2) $\Longrightarrow$ (3) Строим вывод в $\mathbf{S} 5^{\triangleright}$ :

$$
\frac{\bigwedge\{\Pi, \triangleright \Pi, \neg \triangleright \Sigma\} \rightarrow B}{\frac{\bigwedge\{\Pi, \triangleright \Pi, \neg \triangleright \Sigma\} \rightarrow[\triangleright \bigwedge\{\Pi, \triangleright \Pi, \neg \triangleright \Sigma\} \rightarrow \triangleright B]}{\bigwedge\{\Pi, \triangleright \Pi, \neg \triangleright \Sigma, \triangleright \triangleright \Pi, \triangleright \neg \triangleright \Sigma\} \rightarrow \triangleright B}}
$$

$(5) \Longrightarrow(1)$ На $W_{\mathscr{L}}^{\Gamma}$ сначала введем рефлексивное транзитивное отношение $\uparrow$ условием $\left\langle 3{ }_{\mathbf{S}}^{\triangleright}\right\rangle$, а затем возьмем его симметризацию:

$$
w \Uparrow x \leftrightharpoons(w \uparrow x) \&(x \uparrow w) .
$$

Для доказательства $\left\langle 2^{\triangleright}\right\rangle$ возьмем $\left.\Sigma:=\{C|\triangleright C \in| w\rangle\right\}$, а также П и $\Lambda$ как выше. Если бы $\mathscr{L} \vdash B, \Pi, \triangleright(\Pi \Lambda) \Rightarrow \Lambda, \triangleright \Sigma$, то по правилу $(\Rightarrow \mathrm{S} 50$ мы бы вывели $\mathscr{L} \vdash w$. Осталось для такого $x \in W_{\mathscr{L}}^{\Gamma}$, что П, $(\Pi \Lambda) \subseteq\langle x|$ и $\Lambda, \triangleright \Sigma \subseteq|x\rangle$, проверить $w \Uparrow x$. Заметим, что $x \subseteq w$.

Докажем, что $w \uparrow x$. Если $\triangleright C \in\langle w|$, то $C \in \Pi \Lambda$ и $\triangleright C \in\langle x|$. Далее, если $C \in\langle w|$, то $C \in \Pi \subseteq\langle x|$. Если же $C \in|w\rangle$, то $C \in \Lambda \subseteq|x\rangle$.

Докажем, что $x \uparrow w$. Пусть $\triangleright C \in\langle x|$. Тогда $\triangleright C \in w$ ввиду $x \subseteq w$, и если бы $\triangleright C \in|w\rangle$, то $C \in \Sigma$ и $\triangleright C \in|x\rangle$, что неверно; значит, $\triangleright C \in\langle w|$. Далее, если $C \in\langle x|$, то $C \in w$, и если бы $C \in|w\rangle$, то ввиду доказанного включения $\triangleright C \in\langle w|$ имеем $C \in \Lambda \subseteq|x\rangle$, что неверно; поэтому $C \in\langle w|$. Если же $C \in|x\rangle$, то $C \in w$, и если бы $C \in\langle w|$, то ввиду $\triangleright C \in\langle w|$ имеем $C \in \Pi \subseteq\langle x|$, что неверно; поэтому $C \in|w\rangle$.

\section{Логика Grz.}

\footnotetext{
${ }^{2}$ Если в данном применении правила $\left(\Rightarrow \triangleright_{\mathrm{B}}^{0}\right)$ мы могли бы ограничиться условием $\Sigma \Sigma^{\prime} \subseteq$ $\mathrm{Sb}(\Pi \Lambda B)$, то попутно было бы установлено свойство подформульности для исчисления $\left[\mathbf{B}_{2}^{\triangleright}\right]$.
} 
$(2) \Longrightarrow(3)$ Вьводим в $\mathbf{G r z}{ }^{\triangleright}$, используя аксиому $\left(\mathrm{A}_{4}^{\triangleright}\right)$ на последнем шаге:

$$
\frac{\bigwedge\{\Pi, \triangleright \Pi\} \rightarrow(\triangleright(B \rightarrow \triangleright B) \rightarrow B)}{\frac{\bigwedge\{\Pi, \triangleright \Pi\} \rightarrow[\triangleright \bigwedge\{\Pi, \triangleright \Pi\} \rightarrow \triangleright(\triangleright(B \rightarrow \triangleright B) \rightarrow B)]}{\frac{\bigwedge\{\Pi, \triangleright \Pi, \triangleright \triangleright \Pi\} \rightarrow \triangleright B}{\bigwedge\{\Pi, \triangleright \Pi\} \rightarrow \triangleright B .}}}
$$

$(3) \Longrightarrow(4)$ Докажем $\triangleright$-перевод аксиомы $\left(\mathrm{A}_{\mathbf{G}}^{\triangleright}\right)$ в $\operatorname{Grz.}$ С одной стороны, Grz $\vdash \square p \rightarrow p$ и потому

$$
\operatorname{Grz} \vdash(p \rightarrow \triangleright p) \longleftrightarrow(p \rightarrow \square p) .
$$

Отсюда

$$
\begin{aligned}
\text { Grz } \vdash \square \neg(p \rightarrow \triangleright p) \longleftrightarrow \square \neg(p \rightarrow \square p) \\
\longleftrightarrow[\square p \& \square \neg \square p] \longleftrightarrow \neg[\square p \rightarrow \diamond \square p] \longleftrightarrow \perp .
\end{aligned}
$$

Тогда выводим в Grz:

$$
\frac{\frac{\square[\square(p \rightarrow \square p) \rightarrow p] \rightarrow p}{\square[\square(p \rightarrow \triangleright p) \vee \perp \rightarrow p] \rightarrow p}}{\frac{\square[\square(p \rightarrow \triangleright p) \vee \square \neg(p \rightarrow \triangleright p) \rightarrow p] \rightarrow p}{\square[\triangleright(p \rightarrow \triangleright p) \rightarrow p] \rightarrow p}} \frac{\square[(p \rightarrow \triangleright p) \rightarrow p] \rightarrow \square p .}{\square[\triangleright(p)}
$$

С другой стороны,

$$
\operatorname{Grz} \vdash \square \neg[\triangleright(p \rightarrow \triangleright p) \rightarrow p] \longleftrightarrow[\square \triangleright(p \rightarrow \triangleright p) \& \square \neg p] \longrightarrow \square \neg p \longrightarrow \triangleright p
$$

$(5) \Longrightarrow(1)$ Несколько модифицируем метод доказательства, описанньй в п. 3. Допустим, $\mathscr{L} \nvdash \Pi \Rightarrow \Sigma$. Возьмем $\Gamma:=\operatorname{Sb} \Pi \Sigma$,

$$
\widehat{\Gamma}:=\Gamma \cup \operatorname{Sb}\{\triangleright(A \rightarrow \triangleright A), \triangleright(A \vee \triangleright A) \mid \triangleright A \in \Gamma\}
$$

Множество

$$
W_{\mathscr{L}}^{\Gamma}:=\{w \mid w \text { - замкнутая тонкая секвенция, }\langle w|\subseteq \widehat{\Gamma},| w\rangle \subseteq \Gamma, \mathscr{L} \forall w\}
$$

конечно.

ЛЕмма 4.3 (о замыкании). Всякую невыводимую в $\mathscr{L}$ секвенцию $\Pi \Rightarrow \Sigma$ такую, что $\Pi \subseteq \widehat{\Gamma}$ и $\Sigma \subseteq \Gamma$, можно расширить до секвениии из $W_{\mathscr{L}}^{\Gamma}$. Формально, если $\mathscr{L} \nvdash \Pi \Rightarrow \Sigma$, причем $\Pi \subseteq \widehat{\Gamma} u \Sigma \subseteq \Gamma$, mо $\exists w \in W_{\mathscr{L}}^{\Gamma}: \Pi \subseteq\langle w|, \Sigma \subseteq| w\rangle$. 
ДокАЗАТЕльство. В дополнение к доказательству леммы 3.2 надо проверить, что если в процессе замыкания секвенция $\Pi^{\prime} \Rightarrow \Sigma^{\prime}$, невьводимая в $\mathscr{L}$, получена из $\Pi \Rightarrow \Sigma$ добавлением формулы $A \in \mathrm{Sb} \Pi \Sigma, A \notin \Pi \Sigma$, в антецедент или сукцедент, то $\Pi^{\prime} \subseteq \widehat{\Gamma}$ и $\Sigma^{\prime} \subseteq \Gamma$. Первое включение очевидно. При $A \in \Gamma$ второе тоже очевидно. Если же $A \in(\widehat{\Gamma} \backslash \Gamma)$, то ввиду $A \notin \Pi \Sigma$ формула $A$ есть либо $(B \vee \triangleright B)$, либо $(B \rightarrow \triangleright B)$ для некоторой $\triangleright B \in \Pi \Sigma$, причем $\triangleright A \in \Pi$. В обоих случаях $\mathscr{L} \vdash \triangleright A \Rightarrow A$, что следует из леммы $2.3($ б, в), и, значит, $\mathscr{L} \vdash \Pi \Rightarrow \Sigma A$. Поэтому формула $A$ не могла быть добавлена в сукцедент секвенции $\Pi \Rightarrow \Sigma$ и, следовательно, $\Sigma^{\prime}=\Sigma \subseteq \Gamma$.

Как и ранее, для любых $w \in W_{\mathscr{L}}^{\Gamma}$ и $p \in \mathbb{P}$ положим $w \models p \leftrightharpoons p \in\langle w|$. Формулировка и доказательство лемм 3.3 и 3.4 переносятся на наш случай без существенных изменений. На $W_{\mathscr{L}}^{\Gamma}$ введем сначала транзитивное отношение $\uparrow$ условием $\left\langle 3_{\mathrm{S} 4}^{\triangleright}\right\rangle$, далее иррефлексивное транзитивное отношение

$$
w \prec x \leftrightharpoons(w \uparrow x) \&\left(\exists C \in \mathbf{F m}^{\triangleright} \triangleright C \notin\langle w| \& \triangleright C \in\langle x|\right)
$$

и, наконец, рефлексивное транзитивное антисимметричное отношение, т.е. частичньй порядок $w \preccurlyeq x \leftrightharpoons(w \prec x) \vee(w=x)$. Построена конечная Grz-шкала $F_{\mathscr{L}}^{\Gamma}:=\left(W_{\mathscr{L}}^{\Gamma}, \preccurlyeq\right)$. Осталось проверить условие $\left\langle 2^{\triangleright}\right\rangle$, имеющее теперь вид

$$
\forall w \in W_{\mathscr{L}}^{\Gamma} \forall \triangleright B \in w \quad \triangleright B \in\langle w| \Leftrightarrow\left[\begin{array}{ll}
\forall x \succcurlyeq w & B \in\langle x|, \\
\forall x \succcurlyeq w & B \in|x\rangle .
\end{array}\right.
$$

\section{ЛЕмма 4.4. Имеет место импликация $\left\langle 3_{\mathbf{G r z}}^{\triangleright}\right\rangle \Longrightarrow\left\langle 2^{\triangleright}\right\rangle$.}

ДокАЗАтЕльство. Докажем эквивалентность в $\left\langle 2^{\triangleright}\right\rangle$. Возьмем любые $w \in W_{\mathscr{L}}^{\Gamma}$ и $\triangleright B \in w$.

$(\Rightarrow)$ Пусть $\triangleright B \in\langle w|$. Возможны следующие два случая:

1) $B \in\langle w|$, тогда $\forall x \succcurlyeq w$ имеем: либо $w \prec x, w \uparrow x$ и $B \in\langle x|$ по $\left\langle 3_{\mathrm{S} 4}^{\triangleright}\right\rangle$, либо $x=w$ и $B \in\langle w|=\langle x|$;

2) $B \in|w\rangle$, аналогично $\forall x \succcurlyeq w$ получаем $B \in|x\rangle$.

$(\Leftrightarrow)$ Пусть $\triangleright B \in|w\rangle$. Возьмем П, $\Lambda$ как в доказательстве леммы 4.2 .

Случай $B \in|w\rangle$. Положим $y:=w$. Далее имеем

$$
\mathscr{L} \nvdash B, \triangleright(B \vee \triangleright B), \Pi, \triangleright(\Pi \Lambda) \Rightarrow \Lambda,
$$

иначе по правилу $(\underset{\mathbf{G r z}}{\triangleright} \mathbf{0})$ и правилам ослабления получим $\mathscr{L} \vdash w$. Поскольку $\triangleright B \in$ $|w\rangle \subseteq \Gamma$, антецедент вьписанной секвенции содержится в $\widehat{\Gamma}$, а сукцедент в $\Gamma$. По лемме о замькании эту секвенцию можно погрузить в некоторую секвенцию $x \in W_{\mathscr{L}}^{\Gamma}$. Остается проверить,что $w \prec x$. Условие $w \uparrow x$ проверяется как в случае логики $\mathbf{S 4}$. Далее, $\triangleright(B \vee \triangleright B) \in\langle x|$. Но $\triangleright(B \vee \triangleright B) \notin\langle w|$, иначе, учитьвая $B, \triangleright B \in|w\rangle$, мы в силу леммы 2.3 (в) получим даже [ $\left.\mathbf{T}_{2}^{\triangleright}\right]-\vdash w$.

Случай $B \in\langle w|$. Теперь $x:=w$ и аналогично имеем

$$
\mathscr{L} \nvdash \triangleright(B \rightarrow \triangleright B), \Pi, \triangleright(\Pi \Lambda) \Rightarrow \Lambda, B .
$$

Как и выше, погружаем эту секвенцию в некоторьй $y \in W_{\mathscr{L}}^{\Gamma}$. Очевидно, $w \uparrow y$. Наконец, $w \prec y$, поскольку $\triangleright(B \rightarrow \triangleright B) \in\langle y|$, но $\triangleright(B \rightarrow \triangleright B) \notin\langle w|$ ввиду леммы 2.3 (б). 
Теорема полностью доказана.

Напомним, что в присутствии рефлексивности оператор $\square$ выражается через $\triangleright$ посредством равенства $\square p=p \& \triangleright p$. Исходя из этого, введем перевод $\operatorname{Tr}: \mathbf{F m}^{\square} \rightarrow \mathbf{F m}^{\triangleright}$, сохраняющий переменные и булевы связки, а на формулах вида $\square A$ действующий следуюшим образом:

$$
\operatorname{Tr}(\square A)=\operatorname{Tr}(A) \& \triangleright \operatorname{Tr}(A) .
$$

Далее, для произвольной $\triangleright$-логики $M$ обозначим

$$
M^{\square}:=\left\{A \in \mathbf{F m}^{\square} \mid \operatorname{Tr}(A) \in M\right\}=\operatorname{Tr}^{-1}(M) .
$$

Легко видеть что переводы $\operatorname{tr}$ и $\operatorname{Tr}$ взаимно обратны в следующем смысле: $\mathbf{T} \vdash \operatorname{tr}(\operatorname{Tr}(\square p))$ $\leftrightarrow \square p$ и $\mathbf{T}^{\triangleright} \vdash \operatorname{Tr}(\operatorname{tr}(\triangleright p)) \leftrightarrow \triangleright p$. Как следствие, $\left(L^{\triangleright}\right)^{\square}=L$ для любой $\square$-логики $L$, содержащей аксиому $\left(\mathrm{A}_{\mathrm{T}}^{\triangleright}\right)$, а также $\left(M^{\square}\right)^{\triangleright}=M$ для любой $\triangleright$-логики $M$, содержашей аксиому $\left(\mathrm{A}_{\neg}^{\triangleright}\right)$. Отсюда вытекает, что условие $L^{\triangleright}=M$ равносильно конъюнкции условий $\left[M \subseteq L^{\triangleright}\right.$ и $\left.L \subseteq M^{\square}\right]$. Последнее утверждение позволяет строить аксиоматику логики разрешимости над любой нормальной логикой, содержашей $\mathbf{T}$.

ЛЕмма 4.5. Пусть нормальная логика $L$ аксиоматизирована над $\mathbf{T}$ множеством аксиом $\Gamma \subseteq \mathbf{F m}^{\square}$. Тогда логика разрешимости над L имеет следующую аксиоматику:

$$
L^{\triangleright}=\mathbf{T}^{\triangleright}+\operatorname{Tr}(\Gamma), \quad \text { əде } \operatorname{Tr}(\Gamma):=\{\operatorname{Tr}(A) \mid A \in \Gamma\} .
$$

Применяя эту лемму, легко проверить, что $\mathbf{S 4 . 1 ^ { \triangleright }}=\mathbf{S} \mathbf{4}^{\triangleright}+\left(\mathrm{A}_{\mathbf{1}}^{\triangleright}\right)$, где $\left(\mathrm{A}_{\mathbf{1}}^{\triangleright}\right)$ есть аксиома $\triangleright \triangleright p \rightarrow \triangleright p$. Наконец, покажем, что переход $L \mapsto L^{\triangleright}$ является инъективньм гомоморфизмом решетки расширений логики $\mathbf{T}$.

Лемма 4.6. Если $\square$-логики $L, M$ содержсат $\mathbf{T}$, mo

$$
L \subset M \Leftrightarrow L^{\triangleright} \subset M^{\triangleright} \text {. }
$$

ДокАЗАТЕЛЬСтво. Достаточно проверить сохранение нестрогого включения. Из $L \subseteq M$ следует $L^{\triangleright} \subseteq M^{\triangleright}$. Обратно, если $L^{\triangleright} \subseteq M^{\triangleright}$, то $L=\left(L^{\triangleright}\right)^{\square} \subseteq\left(M^{\triangleright}\right)^{\square}=M$.

5. Неустранимость сечения и интерполяция. Здесь мы установим, что во всех построенных в п. 2 секвенциальных исчислениях $\left[L_{k}^{\triangleright}\right], k=1,2$, сечение не устранимо, но в то же время логики $L^{\triangleright}$ обладают интерполяционньм свойством Крейга.

Теорема 5.1. В исиисления $x\left[L_{k}^{\triangleright}\right]$, әде $L \in\{\mathbf{T}, \mathbf{S 4}, \mathbf{S 5}, \mathbf{B}, \mathbf{G r z}\}, k=1,2$, сечение не устранимо.

ДоказАТЕЛьство. 1) Секвенция $\triangleright(p \rightarrow \triangleright p), p \Rightarrow \triangleright p$ выводима в $\left[\mathbf{T}_{2}^{\triangleright}\right]^{-}$(см. лемму $2.3(б))$, а значит, и во всех рассматриваемых исчислениях $\left[L_{k}^{\triangleright}\right]$. Покажем, что она не вьводима без сечения в $\left[\mathbf{B}_{1}^{\triangleright}\right]$ и в $\left[L_{k}^{\triangleright}\right]$ при $L \neq \mathbf{B}$.

Допустим, существует ее вьвод без сечений в одном из этих исчислений. Последним применением неструктурного правила в этом вьводе могло быть только применение одного из правил $(\stackrel{\triangleright}{\neg}),(\Rightarrow \triangleright ⿱ \triangleright 口),\left(\Rightarrow_{L}^{\triangleright}\right)$ или $\left(\Rightarrow_{L}^{\triangleright r}\right)$, при этом первые два исключаются сразу, ибо формулы вида $\triangleright \neg A$ наследуются в выводах без сечений, а в нашей секвенции нет таких формул или подформул. Заключение этого применения должно иметь вид

$$
[\triangleright(p \rightarrow \triangleright p)]^{l},[p]^{m} \Rightarrow[\triangleright p]^{n}, \quad l, m, n \geqslant 0,
$$


поскольку далее в этом вьводе применялись лишь правила ослабления и сокращения. Легко видеть, что при $L \neq \mathbf{B}$ заключения правил $\left(\Rightarrow_{L}^{\triangleright}\right)$ и $\left({ }_{L}^{\triangleright r}\right)$ могут иметь данньй вид лишь при $l=m=0$ и $n>0$. Однако из семантических соображений (используя доказанную теорему о полноте) ясно, что секвенция $\Rightarrow[\triangleright p]^{n}$ не выводима в рассматриваемых исчислениях.

2) Покажем, что секвенция $\triangleright p \Rightarrow \triangleright \neg p$ вьводима даже в $\left[\mathbf{T}_{2}^{\triangleright}\right]^{-}$, но не выводима в $\left[\mathbf{B}_{2}^{\triangleright}\right]$ без сечений. Имеем вьвод в $\left[\mathbf{T}_{2}^{\triangleright}\right]^{-}$:

$$
\begin{gathered}
\frac{p \Rightarrow p}{\Rightarrow p, \neg p} \quad \frac{p \Rightarrow p}{p, \neg p \Rightarrow} \\
\frac{\unrhd_{p \Rightarrow p, \triangleright \neg p}}{\triangleright p, \triangleright p \Rightarrow \triangleright \neg p} \\
\triangleright p \Rightarrow \triangleright \neg p .
\end{gathered}
$$

Допустим, существует ее вывод без сечений в $\left[\mathbf{B}_{2}^{\triangleright}\right]$. Последним применением неструктурного правила в этом вьводе могло быть только применение правила $(\Rightarrow \underset{\mathrm{B}}{\mathrm{r}})$. Его заключение имеет вид: $[\triangleright p]^{m} \Rightarrow[\triangleright \neg p]^{n}$, где $m, n \geqslant 0$. Сопоставляя эту секвенцию с обозначениями из формулировки правила $\left(\Rightarrow_{\mathrm{B}}^{\mathrm{r}}\right)$, имеем: $\Pi=\Lambda=\varnothing, \Sigma^{\prime}=[\triangleright p]^{m}$, $\Sigma=[\triangleright \neg p]^{n-1}$. Посылка этого применения, отвечающая разбиению $\Phi=\Phi^{\prime}=\varnothing$, $\Psi=\Sigma$ и $\Psi^{\prime}=\Sigma^{\prime}$, будет иметь вид

$$
p^{\bar{r}} \Rightarrow p^{r},[\triangleright \triangleright p]^{m},[\triangleright \triangleright \neg p]^{n}, \quad \text { где } r \in\{0,1\} .
$$

Покажем, что последняя секвенция не вьводима в $\left[\mathbf{B}_{2}^{\triangleright}\right]$. В противном случае по теореме о полноте $\mathbf{B}^{\triangleright} \vdash p^{\bar{r}} \rightarrow\left(p^{r} \vee \triangleright \triangleright p \vee \triangleright \triangleright \neg p\right)$. Пусть $r=0$ (случай $r=1$ рассматривается аналогично). Тогда по аксиоме $\left(\mathrm{A}_{\neg}^{\triangleright}\right)$ и правилу $(\mathrm{RE})$ получаем $\mathbf{B}^{\triangleright} \vdash p \rightarrow \triangleright \triangleright p$. Подставив $\neg p$ вместо $p$, мы выведем $\mathbf{B}^{\triangleright} \vdash \neg p \rightarrow \triangleright \triangleright p$. Отсюда $\mathbf{B}^{\triangleright} \vdash \triangleright \triangleright p$, т.е. $\mathbf{B}^{\triangleright}=\mathbf{S 5}^{\triangleright}$, но по лемме 4.5 включение $\mathbf{B}^{\triangleright} \subset \mathbf{S} 5^{\triangleright}$ является строгим.

ОПРЕДЕЛЕНИЕ 5.2. Логика $L$ обладает (интерполяционным) свойством Kрейга, если из $L \vdash A \rightarrow C$ следует существование такой формулы $B$ (интерполянта), что

$$
L \vdash A \rightarrow B, \quad L \vdash B \rightarrow C \quad \text { и } \quad \operatorname{Var} B \subseteq(\operatorname{Var} A \cap \operatorname{Var} C) .
$$

Лемма 5.3. Логика $L \subseteq \mathbf{F m}^{\square}$, содержашая $\mathbf{T}$, обладает свойством Крейга тогда и только тогда, когда логика $L^{\triangleright}$ обладает им.

ДокАЗАТЕЛЬСтво. $(\Rightarrow)$ Воспользуемся очевидной выводимостью $\mathbf{T} \vdash A \leftrightarrow \operatorname{tr}(\operatorname{Tr}(A))$ для всякой $\square$-формулы $A$. Пусть $L^{\triangleright} \vdash A \rightarrow C$, т.е. $L \vdash \operatorname{tr}(A) \rightarrow \operatorname{tr}(C)$. По свойству Крейга для $L$ имеем

$$
\exists B \in \mathbf{F m}^{\square}: \operatorname{Var} B \subseteq(\operatorname{Var} A \cap \operatorname{Var} C), \quad L \vdash \operatorname{tr}(A) \rightarrow B, \quad B \rightarrow \operatorname{tr}(C) .
$$

Тогда $L \vdash \operatorname{tr}(A) \rightarrow \operatorname{tr}(\operatorname{Tr}(B)), \operatorname{tr}(\operatorname{Tr}(B)) \rightarrow \operatorname{tr}(C)$. Отсюда $L \triangleright \vdash A \rightarrow \operatorname{Tr}(B), \operatorname{Tr}(B) \rightarrow C$. Таким образом, $\operatorname{Tr}(B)$ является интерполянтом $A \rightarrow C$ в $L^{\triangleright}$.

$(\Leftarrow)$ Провести те же рассуждения, поменяв ролями переводы $\operatorname{Tr}$ и $\mathrm{tr}$. 
СлЕДСТВИЕ 5.4. Логики $L^{\triangleright}, L \in\{\mathbf{T}, \mathbf{S 4}, \mathbf{B}, \mathbf{S 5}, \mathbf{G r z}, \mathbf{S 4 . 1}\}$, обладают интерполяиионным свойством Крейга.

ДоКАЗАТЕЛЬСТво следует из известного (см. [17], [14]) свойства Крейга для $L$ и леммы 5.3.

Автор благодарит проф. С.Н. Артёмова за помощь при изучении данной темы, а также В. Б. Шехтмана за интерес к работе и полезные дискуссии.

\section{СПИСОК ЦИТИРОВАННОЙ ЛИТЕРАТУРЫ}

[1] Brogan A. P. Aristotle's logic of statements about contingency // Mind. 1967. V. 76. P. $49-81$.

[2] Montgomery H., Routley R. Contingency and non-contingency bases for normal modal logics // Logique et Analyse. 1966. V. 9. P. 318-328.

[3] Montgomery H., Routley R. Noncontingency axioms for $S 4$ and $S 5$ // Logique et Analyse. 1968. V. 11. P. 422-424.

[4] Montgomery H., Routley R. Modalities in a sequence of normal non-contingency modal systems // Logique et Analyse. 1969. V. 12. P. 225-227.

[5] Mortensen C. A sequence of normal modal systems with non-contingency bases // Logique et Analyse. 1976. V. 19. P. 341-344.

[6] Cresswell M. J. Necessity and contingency // Studia Logica. 1988. V. 47. P. 145-149.

[7] Humberstone I. L. The logic of non-contingency // Notre Dame J. Formal Logic. 1995. V. 36. № 2. P. 214-229.

[8] Kuhn S. T. Minimal non-contingency logic // Notre Dame J. Formal Logic. 1995. V. 36. № 2. P. 230-234.

[9] Zolin E. Epistemic non-contingency logic // Notre Dame J. Formal Logic. 1999. V. 40. № 4. P. 533-547.

[10] Золин Е. Секвенциальная логика арифметической разрешимости // Вестн. МГУ. Сер. 1. Матем., мех. 2001. №6. С. 43-48.

[11] Минц Г. Е. Системы Льюиса и система T (1965-1973) // Р. Фейс, Модальная логика. М.: Наука, 1974. С. 423-509.

[12] Ohnishi M., Matsumoto K. Gentzen method in modal calculi // Osaka Math. J. 1957. V. 9. № 2. P. 113-130; Correction // Osaka Math. J. 1958. V. 10. №1. P. 147.

[13] Ohnishi M., Matsumoto K. Gentzen method in modal calculi II // Osaka Math. J. 1959. V. 11. № 2. P. 115-120.

[14] Takano M. Subformula property as a substitute for cut elimination in modal propositional logics // Math. Japonica. 1992. V. 37. №6. P. 1129-1145.

[15] Smullyan R. M. Analytic cut // J. Symbolic Logic. 1968. V. 33. P. 560-564.

[16] Boolos G. The Logic of Provability. Cambridge: Cambridge Univ. Press, 1993.

[17] Chagrov A., Zakharyaschev M. Modal Logic: Oxford Sci. Publ., 1997.

Московский государственный университет им. М.В. Ломоносова Поступило E-mail: zolin@lpcs.math.msu.ru, zolin@mccme.ru 